\title{
Cardiac remodelling and dysfunction in children with obstructive sleep apnoea: a community based study
}

\author{
J Y S Chan, ${ }^{1}$ A M Li, ${ }^{2}$ C-T Au, ${ }^{2}$ A F C Lo, ${ }^{2}$ S-K Ng, ${ }^{3}$ V J Abdullah, ${ }^{3}$ C Ho, ${ }^{4}$ C-M Yu, \\ T-F Fok, ${ }^{2}$ Y-K Wing ${ }^{4}$
}

${ }^{1}$ Division of Cardiology,

Department of Medicine and Therapeutics, Prince of Wales and Shatin Hospital, The Chinese University of Hong Kong, Hong Kong; ${ }^{2}$ Department of Paediatrics, Prince of Wales and Shatin Hospital, The Chinese University of Hong Kong, Hong Kong: ${ }^{3}$ Department of Otorhinolaryngology, Prince of Wales and Shatin Hospital, The Chinese University of Hong Kong, Hong Kong; ${ }^{4}$ Department of Psychiatry, Prince of Wales and Shatin Hospital, The Chinese University of Hong Kong, Hong Kong

Correspondence to: Dr J Y S Chan, Division of Cardiology, Department of Medicine and Therapeutics, Prince of Wales Hospital, The Chinese University of Hong Kong, Hong Kong; jyschan@ cuhk.edu.hk

J Y S Chan and A M Li are joint first authors.

Received 18 December 2007 Accepted 29 September 2008 Published Online First 13 November 2008

\begin{abstract}
Background: Childhood obstructive sleep apnoea (OSA) is suggested to be associated with cardiac structural abnormalities and dysfunction but existing evidence is limited and the treatment effect on echocardiographic outcome remains controversial.

Objective: To examine the presence of subclinical cardiac abnormalities in childhood OSA and the effects of treatment on cardiac changes.
\end{abstract}

Methods: Polysomnography (PSG) and echocardiographic examinations were performed in 101 children aged between 6 and 13 years who were invited from a community based questionnaire survey. They were classified into a reference group (apnoea-hypopnoea index $(A H I)<1, n=35$ ), mild OSA group (AHI 1-5, $\mathrm{n}=39$ ) and moderate to severe group (AHI $>5, \mathrm{n}=27$ ) based on the PSG results. Treatments, including adenotonsillectomy or nasal steroids, were offered to the mild and moderate to severe OSA groups.

Results: The moderate to severe OSA group had greater right ventricular (RV) systolic volume index (RVSVI), lower RV ejection fraction (RVEF) and higher RV myocardial performance index (RVMPI) than the reference group. They also had more significant left ventricular (LV) diastolic dysfunction and remodelling with larger interventricular septal thickness index (IVSI) and relative wall thickness than those with lower AHI values. The moderate to severe OSA group had an increased risk of abnormal LV geometry compared with the reference group (odds ratio 4.21 (95\% $\mathrm{Cl} 1.35$ to 13.12)). Log transformed AHI was associated with RVSVI $(p=0.0002)$, RVEF $(p=0.0001)$ and RVMPI $(p<0.0001)$, independent of the effect of obesity. Improvement in RVMPI, IVSI and E/e' were observed in those with a significant reduction in $\mathrm{AHI}(>50 \%)$ comparing 6 month with baseline data.

Conclusions: OSA is an independent risk factor for subclinical RV and LV dysfunction, and improvement in $\mathrm{AHI}$ is associated with reversibility of these abnormalities.

Childhood obstructive sleep apnoea (OSA) is being increasingly recognised and its prevalence among the paediatric population is reported as $2-4 \% .{ }^{1}$ Early studies on childhood OSA demonstrated that severe disease could lead to congestive heart failure. ${ }^{23}$ More recent reports have documented the presence of subclinical forms of cardiac/ ventricular dysfunction in children with OSA. ${ }^{47}$ However, these paediatric studies were limited by their small sample size and lack of normal children as controls. All cases included in these studies were recruited from hospital attendants which may not truly reflect the situation in the community. Furthermore, the effect of intervention for OSA on cardiac dysfunction has not been well characterised. Preventing the development of subclinical cardiac abnormalities may be important as current evidence suggests asymptomatic ventricular dysfunction predicts future cardiovascular events. ${ }^{89}$ The aims of this study were to assess cardiac structure and function in an OSA cohort recruited from the community and to measure the degree of reversibility following interventions.

\section{METHODS}

\section{Subjects and study design}

Subjects for this study were drawn from our ongoing childhood OSA epidemiological study which involved children aged between 6 and 13 years recruited from 13 randomly chosen schools. A total of 6447 schoolchildren had completed a validated OSA screening questionnaire ${ }^{10}$ that stratified them into high $(n=586)$ or low $(n=5861)$ risk for OSA. All children classified as high risk by the questionnaire were invited, and subjects classified as low risk were randomly chosen and invited for overnight polysomnography (PSG) and cardiac assessment. A total of 410 high risk and 209 low risk children agreed to participate. Children were excluded from the study if they had an intercurrent upper respiratory tract infection within 4 weeks of PSG, suffered from neuromuscular disorder, craniofacial anomalies, syndromic disorder or if they had previously undergone upper airway surgery. Anthropometric parameters, including weight, height, waist and hip circumferences, and casual systolic and diastolic blood pressure were measured (Datascope Accutorr Plus) on the day of PSG. Two blood pressure measurements were taken with a 5 min interval and the average of the two readings was used for analysis. Body mass index (BMI) was translated to BMI $z$ scores according to the normal reference values of Hong Kong Chinese children. ${ }^{11}$ This project included the first 101 consecutive children who gave consent for overnight PSG and echocardiographic examination (ECHO).

All children were referred for otorhinolaryngological assessment before their PSG. For those who were diagnosed as having OSA based on PSG, adenotonsillectomy was offered. Those who refused surgical intervention or where surgery was thought not to be indicated based on predetermined criteria (small tonsils; tonsils do not extend beyond the anterior tonsillar pillar and small adenoids; adenoids which occupy less than $25 \%$ of the post-nasal space with minimal OSA symptoms or poorly controlled allergic rhinitis with supine nasal obstruction) were offered nasal 
Figure 1 Flow diagram: 101 subjects consented to undergo polysomnography (PSG) and echocardiography (ECHO), of which 35 were controls, 39 had mild obstructive sleep apnoea (OSA) and 27 had moderate to severe OSA. Twenty patients in the mild OSA group and 10 in the moderate to severe OSA group refused follow-up. The remaining patients underwent adenotonsillectomy, had nasal steroid therapy or were left untreated because they refused treatment. At follow-up, in 17 their apnoea-hypopnoea index (AHI) had decreased by more than $50 \%$.

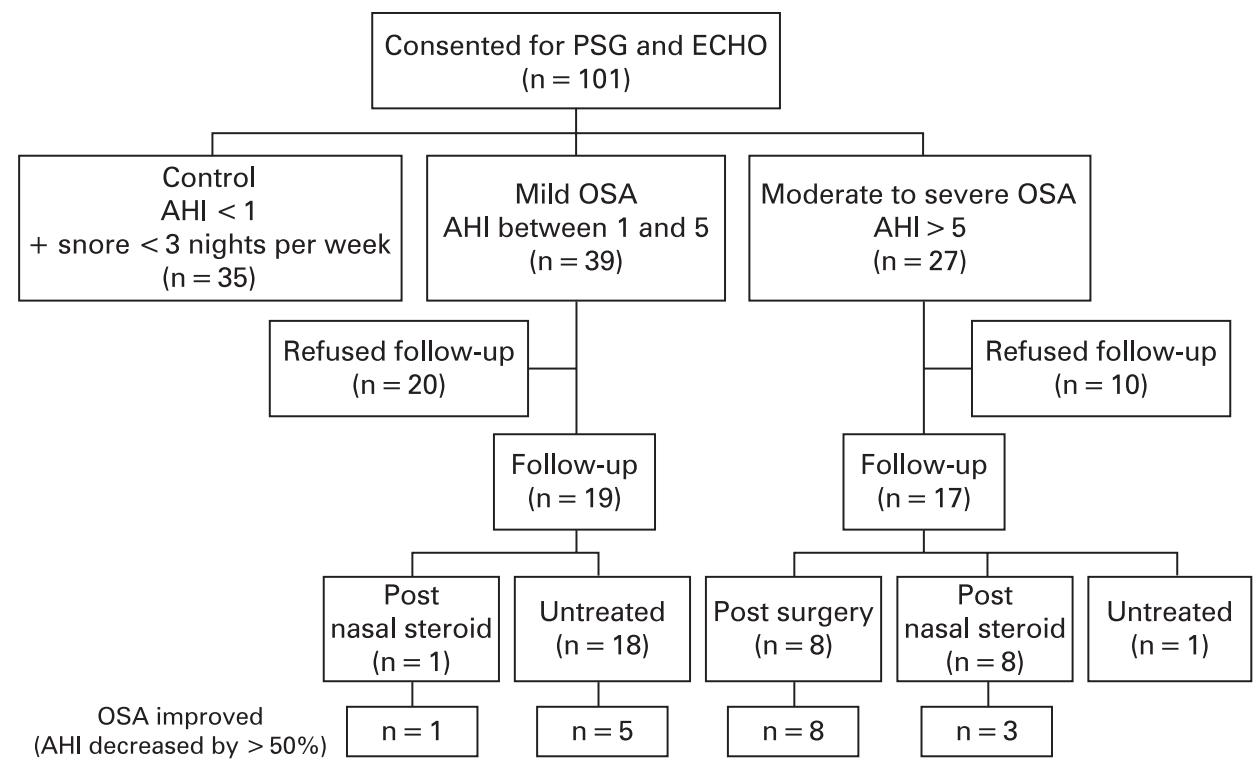

corticosteroid therapy (mometasone $100 \mu \mathrm{g} /$ day for 6 months) ${ }^{12}$ and/or non-invasive positive pressure ventilation. These subjects then underwent repeat PSG and ECHO 6 months after the operation or start of therapy. Children with OSA who refused any form of therapy were also invited to return at 6 months for a repeat assessment. Informed consent from the parents or legal guardian and verbal assent from each child were obtained. The study was approved by the institutional ethics committee.

\section{Polysomnography}

Children recruited into the study underwent at least one standard overnight PSG at a dedicated sleep laboratory with CNS 1000P polygraph (CNS; Chanhassen Minnesota, USA). The central and occipital electroencephalogram, bilateral electrooculogram, submental electromyogram, bilateral leg electromyogram and electrocardiogram were recorded. The positions of the subject, respiratory airflow (nasal cannula connected to pressure transducer), respiratory efforts (strain gauge), arterial oxyhaemoglobin saturation $\left(\mathrm{SpO}_{2}\right.$, Ohmeda 3700 pulse oximeter, measured by finger sensor, averaging time $3 \mathrm{~s}$ ) were measured. In this study, we scored the presence of both apnoeas and hypopnoeas. Briefly, obstructive apnoea was defined as absence of airflow with persistent respiratory effort lasting longer than two baseline breaths, irrespective of $\mathrm{SpO}_{2}$ changes. Obstructive hypopnoea was defined as reduction of airflow of $50 \%$ or more with persistent respiratory effort lasting longer than two baseline breaths and associated with oxygen desaturation of at least $4 \%$ and/or arousals. The apnoeahypopnoea index (AHI) was the total number of obstructive apnoeas or hypopnoeas per hour of sleep. Oxygen desaturation index (ODI) was defined as the total number of dips in arterial oxygen saturation $\geqslant 4 \%$ per hour of sleep. Arousal was defined

Table 1 Demographic, anthropometric and polysomnography characteristics

\begin{tabular}{|c|c|c|c|c|c|}
\hline & $\begin{array}{l}\text { Total } \\
(\mathrm{n}=101)\end{array}$ & $\begin{array}{l}\text { Reference } \\
(\mathrm{n}=35)\end{array}$ & $\begin{array}{l}\text { Mild OSA } \\
(\mathrm{n}=39)\end{array}$ & $\begin{array}{l}\text { Moderate to } \\
\text { severe OSA } \\
(\mathrm{n}=27)\end{array}$ & $\mathrm{p}$ Value \\
\hline \multicolumn{6}{|l|}{ Demographics and anthropometrics } \\
\hline Age (year) & $9.6(1.9)$ & $9.5(1.9)$ & $9.7(1.9)$ & $9.6(1.8)$ & 0.9 \\
\hline Male gender $(\mathrm{n}(\%))$ & $74(73.3)$ & $25(71.4)$ & $25(64.1)$ & $24(88.9)$ & 0.08 \\
\hline Body height $(\mathrm{cm})$ & $135.2(12.3)$ & $132.9(11.3)$ & $135.7(12.1)$ & $137.3(13.6)$ & 0.3 \\
\hline Body weight $(\mathrm{kg})$ & $35.7(11.6)$ & $33.7(10.6)$ & $34.8(11.3)$ & $39.6(12.7)$ & 0.1 \\
\hline Body mass index $\left(\mathrm{kg} / \mathrm{m}^{2}\right)$ & $19.1(3.7)$ & $18.7(3.6)$ & $18.4(3.7)$ & $20.4(3.5)$ & 0.06 \\
\hline Body mass index z score $\ddagger$ & $0.8(1.0)$ & $0.8(1.1)$ & $0.60(1.1)$ & $1.24(0.8)$ & 0.04 \\
\hline Body surface area $\left(\mathrm{m}^{2}\right)$ & $1.1(0.2)$ & $1.1(0.2)$ & $1.1(0.2)$ & $1.2(0.3)$ & 0.2 \\
\hline Waist-to-hip ratio & $0.9(0.1)$ & $0.9(0.1)$ & $0.9(0.1)$ & $0.9(0.1)$ & 0.2 \\
\hline Systolic blood pressure $(\mathrm{mm} \mathrm{Hg})$ & $104.7(11.7)$ & $102.6(11.9)$ & $104.2(10.5)$ & $108.2(12.8)$ & 0.2 \\
\hline Diastolic blood pressure $(\mathrm{mm} \mathrm{Hg})$ & $68.0(9.7)$ & $67.3(9.7)$ & $66.9(10.5)$ & $70.6(8.4)$ & 0.3 \\
\hline \multicolumn{6}{|l|}{ Polysomnographics } \\
\hline Sleep efficiency (\%) & $81.1(11.2)$ & $82.3(8.7)$ & $80.3(11.0$ & $80.8(14.2$ & 0.7 \\
\hline Apnoea-hypopnoea index $/ \mathrm{h}^{*} \dagger$ t & $1.9(0.5-5.5)$ & $0(0-0.6)$ & $2.1(1.4-3.0)$ & $10.2(6.9-17.3)$ & $<0.0001$ \\
\hline Oxygen desaturation index $/ \mathrm{h}^{*} \dagger+$ & $0.4(0-1.6)$ & $0(0-0.2)$ & $0.5(0.2-1.1)$ & $4.0(2.3-9.5)$ & $<0.0001$ \\
\hline Arousal index/h†t & $6.8(5.1-9.5)$ & $5.8(3.7-7.2)$ & $6.0(4.9-8.0)$ & $10.3(8.0-14.9)$ & $<0.0001$ \\
\hline Oxyhaemoglobin saturation nadir $(\%)^{*} \uparrow$ & $91.3(3.9)$ & $93.6(2.2)$ & $91.4(1.9)$ & $88.1(5.3)$ & $<0.0001$ \\
\hline
\end{tabular}

Parametric data and non-parametric data are shown as mean (SD) and median (IQR), respectively.

${ }^{*} \mathrm{p}<0.05$, normal group vs mild OSA group. $\dagger \mathrm{p}<0.05$, normal group vs moderate to severe OSA group. $\$ p<0.05$, mild 0SA group vs moderate to severe OSA group.

OSA, obstructive sleep apnoea. 
Table 2 Group comparisons of right and left ventricular structure and function

\begin{tabular}{|c|c|c|c|c|c|}
\hline & $\begin{array}{l}\text { Total } \\
(n=101)\end{array}$ & $\begin{array}{l}\text { Reference } \\
(n=35)\end{array}$ & $\begin{array}{l}\text { Mild OSA } \\
(\mathrm{n}=39)\end{array}$ & $\begin{array}{l}\text { Moderate to } \\
\text { severe OSA } \\
(\mathrm{n}=27)\end{array}$ & p Value \\
\hline \multicolumn{6}{|l|}{ Right ventricle (RV) } \\
\hline RV systolic volume index $(\mathrm{ml} / \mathrm{m})^{*} \dagger$ & $0.23(0.09)$ & $0.18(0.08)$ & $0.26(0.10)$ & $0.25(0.07)$ & $<0.001$ \\
\hline RV ejection fraction $(\%)^{*} \dagger$ & $53.4(12.5)$ & $59.3(11.0)$ & $51.4(12.3)$ & $48.7(11.9)$ & 0.001 \\
\hline $\mathrm{RV}$ myocardial performance index $\dagger$ & $0.37(0.12)$ & $0.32(0.09)$ & $0.36(0.09)$ & $0.46(0.14)$ & $<0.001$ \\
\hline \multicolumn{6}{|l|}{ Left ventricle (LV) } \\
\hline LV systolic volume index $(\mathrm{ml} / \mathrm{m})$ & $22.6(9.6)$ & $23.0(9.8)$ & $23.0(8.9)$ & $21.4(10.5)$ & NS \\
\hline LV ejection fraction (\%) & $66.2(10.7)$ & $66.3(10.1)$ & $64.4(10.0)$ & $68.6(12.1)$ & NS \\
\hline LV mass index $\left(\mathrm{g} / \mathrm{m}^{2.7}\right)$ & $32.6(9.5)$ & $32.5(7.8)$ & $30.3(7.3)$ & $36.3(12.9)$ & 0.04 \\
\hline Relative wall thickness: & $0.36(0.07)$ & $0.36(0.07)$ & $0.34(0.06)$ & $0.39(0.07)$ & 0.005 \\
\hline Interventricular septal thickness index $(\mathrm{cm} / \mathrm{m})$ & $0.56(0.09)$ & $0.56(0.09)$ & $0.52(0.09)$ & $0.60(0.09)$ & 0.004 \\
\hline LV posterior wall thickness index $(\mathrm{cm} / \mathrm{m})$ & $0.52(0.10)$ & $0.52(0.10)$ & $0.50(0.09)$ & $0.56(0.11)$ & NS \\
\hline Abnormal LV geometry $(\mathrm{n}(\%)) \S$ & $43(42.6)$ & $14(40.0)$ & $9(23.1)$ & $20(74.1)$ & $<0.001$ \\
\hline LV myocardial performance index & $0.33(0.11)$ & $0.32(0.08)$ & $0.32(0.12)$ & $0.35(0.14)$ & NS \\
\hline $\mathrm{E} / \mathrm{A}$ (mitral valve) & $2.2(1.6)$ & $2.0(0.6)$ & $2.6(2.5)$ & $2.0(0.4)$ & NS \\
\hline $\mathrm{E} / \mathrm{e}^{\prime} \dagger \mathrm{t}$ & $9.5(1.8)$ & $9.1(1.8)$ & $9.3(1.4)$ & $10.4(2.1)$ & 0.009 \\
\hline
\end{tabular}

as an abrupt shift in electroencephalogram frequency during sleep, which may include theta, alpha and/or frequencies greater than $16 \mathrm{~Hz}$ but not spindles, of $3-15 \mathrm{~s}$ in duration. In REM sleep, arousals were scored only when accompanied by concurrent increases in submental electromyogram amplitude. Arousal index (ArI) was defined as the number of arousals per hour of sleep. OSA was defined if AHI $\geqslant 1$ per hour of sleep. ${ }^{13}$

\section{Echocardiography}

ECHO studies were performed using the Vivid 5 system (Vingmed-General Electric, Horten, Norway). The examiner was blinded to the subject's PSG results and therefore group allocation. Left ventricular (LV) volumes and ejection fraction (EF) were assessed by biplane Simpson's equation using the apical four chamber and two chamber views where the length of the ventricular image was maximised. LV mass was measured by Devereux's method, as previously described. ${ }^{14}$ LV mass was divided by subject's height to the power of 2.7 to provide LV mass index (LVMI). LV hypertrophy was defined as an LVMI greater than the 95 th percentile of healthy controls $\left(38.6 \mathrm{~g} / \mathrm{m}^{2.7}\right)$ that was previously reported. ${ }^{15}$ Relative wall thickness (RWT) of the LV, a measure of concentricity, was calculated as the sum of the thickness of the posterior and septal wall divided by LV diastolic diameter. A RWT of greater than the 95th percentile of age matched healthy controls (0.375) that was previously reported was used as the cut-off to define concentric LV geometry. ${ }^{16}$ LV geometry was classified as normal, concentric remodelling, eccentric hypertrophy or concentric hypertrophy, as described by Ganau and colleagues. ${ }^{17}$ Right ventricular end systolic (RVSVI) and end-diastolic volume index to height (RVDVI) and the right ventricular ejection fraction (RVEF) were calculated from apical four chamber views, using the arealength method (RV volume $=3 / 8 \pi\left[\mathrm{area}^{2} /\right.$ length]). Any significant valvular lesion was examined with haemodynamic assessment made. In children with tricuspid regurgitation, a spectral Doppler profile was used to estimate pulmonary artery systolic pressure from the sum of the modified Bernoulli equation $\left(\right.$ velocity $\left.^{2} \times 4\right)$ product and estimated right atrial pressure. Diastolic function was measured for both ventricles by measuring the ratio of the peak early diastolic (E) and peak atrial (A) velocity from pulse Doppler data from the tricuspid and mitral valves. LV and RV myocardial performance index (MPI) of both ventricles were defined by the sum of isovolumic contraction and relaxation times divided by ejection time obtained from pulse Doppler data. LV filling pressure was approximated from the relationship $\mathrm{E} / \mathrm{e}^{\prime}$ where $\mathrm{E}$ is the pulse wave Doppler velocity of the mitral valve and $e^{\prime}$ is the pulse wave tissue Doppler velocity of the septal annulus.

\section{Statistical analysis}

All parametric and non-parametric data are expressed as mean (SD) and median (IOR), respectively. Parametric and nonparametric data were compared using one way analysis of variance (ANOVA) and the Kruskal-Wallis test, respectively. For parametric data, the Tukey or Games-Howell method was used for post-hoc pairwise comparisons with adjustments made depending on the agreement of the assumption of variance. For non-parametric data, the Mann-Whitney $U$ test with adjusted $p$ values (significant at $p<0.016$ ) were used for pairwise comparisons. The $\chi^{2}$ test was performed to investigate the difference in proportions between groups. Further $\chi^{2}$ tests with adjusted $p$ values $(p<0.016)$ were used for pairwise comparisons.

Multiple linear regression analyses were performed to assess the relationship between PSG variables and ECHO measures, while controlling for possible confounders. As some of the PSG data were skewed and contained zero values, these variables were log transformed using special formula (natural log $[\mathrm{x}+0.1])$.

Logistic regression analyses were performed to estimate the odds ratios (OR) for abnormal LV geometry for different severities of OSA compared with the reference group while adjusting for possible confounding factors.

For the follow-up study, subjects were divided into two groups for comparison. One group consisted of subjects who had improvement in their OSA, as defined by $>50 \%$ reduction in AHI from baseline to follow-up. ${ }^{18}$ The other group consisted of subjects who had persistent or worsened OSA $(<50 \%$ 
Table 3 Multiple linear regression analyses showing the association between polysomnography and echocardiography parameters after adjustment for age, gender and body mass index z score

\begin{tabular}{|c|c|c|c|c|}
\hline \multirow[b]{2}{*}{ Variable } & \multicolumn{2}{|l|}{ Log transformed AHI } & \multicolumn{2}{|l|}{ Log transformed ODI } \\
\hline & $\beta(95 \% \mathrm{Cl})$ & p Value & $\beta(95 \% \mathrm{CI})$ & p Value \\
\hline \multicolumn{5}{|l|}{ Right ventricle } \\
\hline Systolic volume index & $1.6(0.8$ to 2.5$)$ & 0.0002 & $1.2(0.2$ to 2.1$)$ & 0.017 \\
\hline Ejection fraction & $-2.7(-4.0$ to -1.3$)$ & 0.0001 & $-2.0(-3.5$ to -0.5$)$ & 0.009 \\
\hline Myocardial performance index & $0.033(0.021$ to 0.046$)$ & $<0.0001$ & $0.025(0.011$ to 0.040$)$ & 0.0007 \\
\hline \multicolumn{5}{|l|}{ Left ventricle } \\
\hline Left ventricular mass index & $0.31(-0.78$ to 1.39$)$ & NS & $0.62(-0.54$ to 1.78$)$ & NS \\
\hline Relative wall thickness & $0.003(-0.006$ to 0.011$)$ & NS & $0.006(-0.003$ to 0.015$)$ & NS \\
\hline Interventricular septal thickness index & $0.007(-0.004$ to 0.018$)$ & NS & $0.015(0.003$ to 0.026$)$ & 0.016 \\
\hline $\mathrm{E} / \mathrm{e}^{\prime}$ & 0.21 (0 to 0.42$)$ & 0.05 & $0.17(-0.05$ to 0.40$)$ & NS \\
\hline
\end{tabular}

$\mathrm{AHI}$, apnoea-hypopnoea index; $\mathrm{E} / \mathrm{e}^{\prime}$, ratio of mitral early peak velocity/mitral annulus early peak velocity; $\mathrm{ODI}$, oxygen desaturation index.

reduction in $\mathrm{AHI})$. Wilcoxon signed ranks tests were used to examine the intragroup differences between baseline and follow-up. Mann-Whitney $U$ tests were used to detect the between group differences at baseline, at follow-up and the changes during the treatment period. All analyses were performed using SPSS V.13.0.

\section{RESULTS}

\section{Study population}

The children were divided into a reference group (AHI $<1$ and snoring for $<3$ nights per week, $\mathrm{n}=35$ ), mild OSA group (AHI $1-5, \mathrm{n}=39$ ) and moderate to severe OSA group (AHI $>5$, $\mathrm{n}=27$ ) (fig 1). The demographics, anthropometric and PSG data for the groups are shown in table 1, and there were no significant differences in demographic or anthropometric parameters except for BMI z score. Significant differences were found in $\mathrm{AHI}, \mathrm{ODI}$, ArI and $\mathrm{SpO}_{2}$ nadir between the groups.

\section{ECHO findings of cardiac structure and function}

\section{Right ventricle}

RVSVI, RVEF and RVMPI were all significantly different between the three groups. The reference group had a significantly smaller RVSVI and higher EF than both the mild and moderate to severe OSA groups. For RVMPI, the post hoc test showed that the moderate to severe group had a significantly greater value than the mild and reference groups (table 2). No significant tricuspid regurgitation was noted in any of the children.

Multiple linear regression analysis showed that log transformed AHI and log transformed ODI were significantly associated with RVSVI $(p=0.0002), \operatorname{RVEF}(p=0.0001)$ and RVMPI ( $p<0.0001)$ after adjustment for age, gender and BMI $z$ score (table 3).

\section{Left ventricle}

RWT and the interventricular septal thickness index to height (IVSI) were significantly higher in the moderate to severe group compared with the mild group (table 2). A similar trend was observed for LVMI although subgroups comparison showed no significant difference. The moderate to severe group also had a greater proportion of subjects having abnormal LV geometry compared with the mild and reference groups. For diastolic function parameters, $\mathrm{E} / \mathrm{e}^{\prime}$, a marker of LV filling pressure, increased with increasing severity of OSA.

Multiple linear regression analysis showed that log transformed ODI were significantly associated with IVSI $(p=0.016)$ after adjustment for age, gender and BMI $z$ score. The results also showed that the adjusted association between log transformed AHI and $\mathrm{E} / \mathrm{e}^{\prime}$ was nearly significant $(\mathrm{p}=0.050)$ (table 3).

Multivariate logistic regression analyses were used to examine whether the severity of OSA was associated with the presence of abnormal LV geometry. The risk of abnormal LV geometry was 4.29 times (OR 4.29 (95\% confidence interval (CI) 1.43 to 12.81), $p=0.009$ ) higher for the moderate to severe group compared with the reference group before adjusting for any confounders. The OR was reduced but remained significant after adjustment for age, gender and BMI z score (OR 4.21 (95\% CI 1.35 to 13.12), $p=0.013)$. Such an increased risk was not found in the mild OSA group.

\section{Treatment effect}

Thirty out of 66 OSA subjects refused to have follow-up assessment. Of the remaining 36 OSA subjects, eight had adenotonsillectomy and nine received nasal steroid therapy. No child opted to receive non-invasive positive pressure ventilation in our study. The remaining 19 subjects refused any form of treatment but agreed to have follow-up assessment (recruitment details are described in fig 1). Their characteristics, including anthropometrics, PSG and ECHO parameters were not significantly different from those who did not return for reassessment. At follow-up, 17 had improved OSA, as reflected by their AHI decrement of $\geqslant 50 \%$ (improved OSA group). ${ }^{18}$ The remaining $(n=19)$ were classified as persistent OSA group.

Significant reductions in RVMPI, IVSI, and E/e' from baseline to follow-up 6 months later were noted only in the improved OSA group (table 4). Changes in RWT and E/e' from baseline were significantly different between the improved OSA group and the persistent OSA group (table 4). Furthermore, change in AHI was positively associated with changes in RVMPI (Spearman's rho $(r)=0.448, p=0.006)$, IVSI $(r=0.429$, $p=0.009)$, RWT $(r=0.433, p=0.008)$ and $E / e^{\prime} \quad(r=0.502$, $\mathrm{p}=0.002$ ) (fig 2). These changes in ECHO parameters were not significantly associated with change in BMI $z$ score.

\section{DISCUSSION}

In this study, we were able to document RV and LV dysfunction and remodelling in a cohort of community based children with OSA. The AHI was demonstrated to be a significant independent parameter associated with cardiac dysfunction. Following effective treatment for OSA, the cardiac abnormalities improved whereas in the group with persistent OSA, the abnormalities showed no improvement. These 
Table 4 Intra- and intergroup comparisons between the effectively treated group and the ineffectively treated group

\begin{tabular}{|c|c|c|c|}
\hline & $\begin{array}{l}\text { Persistent 0SA } \\
(\Delta A H I \geqslant 50 \%)(n=19)\end{array}$ & $\begin{array}{l}\text { Improved 0SA } \\
(\Delta \mathrm{AHI}<\mathbf{5 0} \%)(\mathrm{n}=\mathbf{1 7})\end{array}$ & p Value \\
\hline Age at baseline (years) & $8.8(7.5,9.4)$ & $9.8(8.2,11.1)$ & NS \\
\hline \multicolumn{4}{|l|}{ Body mass index $z$ score } \\
\hline Baseline & $1.41(0.67,1.78)$ & $0.84(-0.15,1.82)$ & NS \\
\hline Follow-up & $1.63(0.76,1.78)$ & $0.63(-0.20,1.82)$ & NS \\
\hline${ }^{*} p$ Value & NS & NS & \\
\hline Change & $0.04(-0.20,0.22)$ & $0.10(-0.13,0.22)$ & NS \\
\hline \multicolumn{4}{|l|}{ Apnoea-hypopnoea index/h } \\
\hline Baseline & $3.0(2.3,6.9)$ & $10.2(2.6,17.5)$ & NS \\
\hline Follow-up & $6.0(2.8,7.8)$ & $0.8(0.3,3.4)$ & $<0.001$ \\
\hline${ }^{*} p$ Value & NS & 0.0003 & \\
\hline Change & $1.5(-0.5,4.2)$ & $-7.0(-15.3,-2.3)$ & $<0.001$ \\
\hline \multicolumn{4}{|l|}{ RV ejection fraction $(\%)$} \\
\hline Baseline & $51.5(43.4,60.3)$ & $47.2(42.7,58.5)$ & NS \\
\hline Follow-up & $50.5(36.7,58.3)$ & $55.5(45.1,60.4)$ & NS \\
\hline${ }^{*} p$ Value & NS & NS & \\
\hline Change & $-1.9(-9.3,3.5)$ & $1.7(-1.9,12.5)$ & NS \\
\hline \multicolumn{4}{|l|}{ RV myocardial performance index } \\
\hline Baseline & $0.354(0.321,0.414)$ & $0.490(0.408,0.555)$ & 0.011 \\
\hline Follow-up & $0.337(0.288,0.430)$ & $0.354(0.288,0.402)$ & NS \\
\hline${ }^{*} p$ Value & NS & 0.025 & \\
\hline Change & $-0.03(-0.16,0.10)$ & $-0.11(-0.25,0.04)$ & NS \\
\hline \multicolumn{4}{|l|}{ Relative wall thickness } \\
\hline Baseline & $0.338(0.302,0.376)$ & $0.372(0.319,0.464)$ & NS \\
\hline Follow-up & $0.377(0.333,0.418)$ & $0.372(0.311,0.404)$ & NS \\
\hline${ }^{*} p$ Value & NS & 0.039 & \\
\hline Change & $0.014(-0.002,0.074)$ & $-0.017(-0.063,0.001)$ & 0.006 \\
\hline \multicolumn{4}{|c|}{ Interventricular septal thickness index $(\mathrm{cm} / \mathrm{m})$} \\
\hline Baseline & $0.507(0.490,0.633)$ & $0.606(0.500,0.661)$ & NS \\
\hline Follow-up & $0.540(0.440,0.648)$ & $0.528(0.500,0.577)$ & NS \\
\hline${ }^{*} p$ Value & NS & 0.022 & \\
\hline Change & $-0.008(-0.070,0.120)$ & $-0.036(-0.097,-0.008)$ & NS \\
\hline \multicolumn{4}{|l|}{$\mathrm{E} / \mathrm{e}^{\prime}$} \\
\hline Baseline & $9.3(8.7,9.9)$ & $10.2(8.8,12.3)$ & NS \\
\hline Follow-up & $9.4(8.9,11.2)$ & $9.1(8.4,9.9)$ & NS \\
\hline${ }^{*}$ p Value & NS & 0.013 & \\
\hline Change & $0.3(-0.8,1.7)$ & $-1.4(-3.2,-0.1)$ & 0.009 \\
\hline \multicolumn{4}{|l|}{ Abnormal LV geometry, N (\%) } \\
\hline Baseline & $7(36.8)$ & $10(58.8)$ & NS \\
\hline Follow-up & $10(52.6)$ & $8(47.1)$ & NS \\
\hline${ }^{*}$ p Value & NS & NS & \\
\hline Acquired abnormal LV geometry (n (\%)) & $6(31.6)$ & $2(11.8)$ & NS \\
\hline Normalised LV geometry (n (\%)) & $3(15.8)$ & $4(23.5)$ & NS \\
\hline
\end{tabular}

Data are shown as median (IQR) unless otherwise specified.

${ }^{*} p$ Values were obtained from Wilcoxon signed ranks tests (intragroup comparisons).

AHI, apnoea-hypopnoea index; E/e', ratio of mitral early peak velocity/mitral annulus early peak velocity; LV, right ventricle;

OSA, obstructive sleep apnoea; RV, right ventricle.

findings are important because children who are otherwise healthy have end organ involvement with RV and LV structural and functional abnormalities similar to those that have been associated with other diseases, ${ }^{19}{ }^{20}$ such as hypertension. In adults, evidence supports the fact that abnormalities detected in $\mathrm{ECHO}$, even in asymptomatic patients, predict future cardiovascular events. ${ }^{89}$ Regression of these cardiac abnormalities with treatment is associated with a decrease in cardiovascular morbidity, including atrial fibrillation, heart failure and hospitalisation..$^{21} 22$

There are disparate conclusions of previous studies on the effect of OSA on RV function and enlargement. ${ }^{43-25}$ Sanner et al has shown that OSA was independently associated with depressed RVEF by radionuclide ventriculography. ${ }^{23}$ In other studies, RV dimensions and RV systolic function measured by ECHO were not shown to be significantly different between subgroups with varying OSA severity. ${ }^{23}{ }^{24}$ A possible explanation for the discrepancy is that previous studies were in subjects with varying degrees of obesity recruited from hospital attendants. Obesity is an important risk factor of OSA in adults and children, ${ }^{26}$ and it is well established that obesity and its metabolic complications are risk factors for cardiac abnormalities. ${ }^{27}{ }^{28}$ Thus failing to control for obesity could have given conflicting results in the relationship between OSA and RV dysfunction. Our study showed that RVSVI, RVEF and RVMPI worsen with OSA severity, and AHI independently correlated with these parameters, even after adjusting for age, gender and BMI z score. Cor pulmonale was consistently observed in a 
Figure 2 Association between change in apnoea-hypopnoea index (AHI) and changes in echocardiography (ECHO) parameters. The change in $\mathrm{AHI}(\triangle \mathrm{AHI})$ was significantly associated with changes in $(A)$ right ventricular myocardial performance index ( $\triangle \mathrm{RVMPI})$, (B) interventricular septal thickness index ( $\triangle \mathrm{IVSI}),(\mathrm{C})$ relative wall thickness $(\triangle \mathrm{RWT})$ and $(\mathrm{D})$ ratio of mitral early peak velocity/mitral annulus early peak velocity $\left(\Delta \mathrm{E} / \mathrm{e}^{\prime}\right)$.
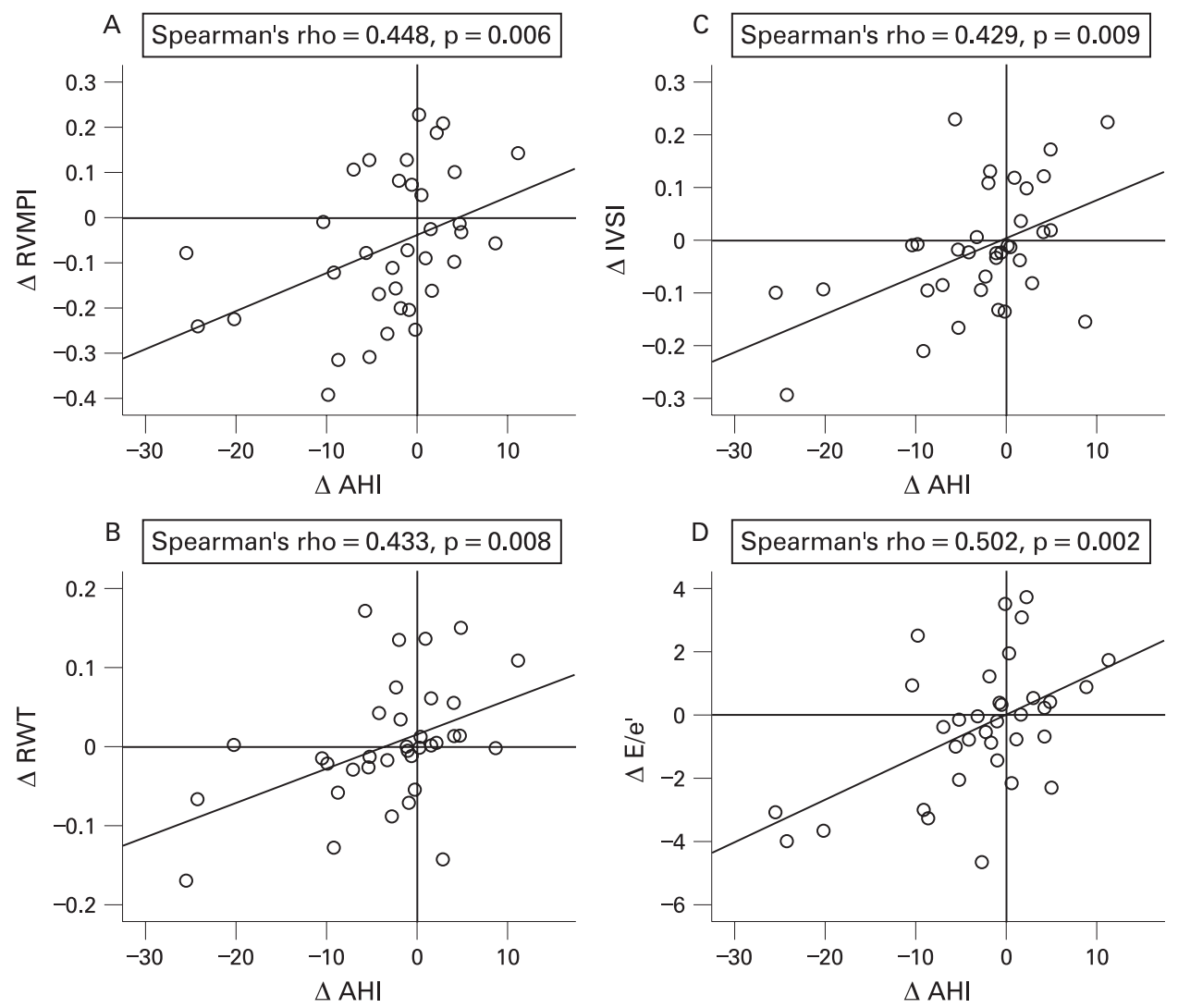

previous study in children with severe OSA. ${ }^{29}$ On the other hand, none of the children in our study had cor pulmonale. Nonetheless, our study provides evidence for the first time that subclinical RV dysfunction already exists in children with mild degrees of OSA. Further evidence to support RV involvement in childhood OSA is that RVMPI significantly improved after interventions, as shown in our study as well as in that reported by $\mathrm{Tal}$ and colleagues. ${ }^{4}$ Moreover, the change in RVMPI from baseline to follow-up was significantly associated with the change in AHI (fig 2).

After controlling for age, gender and BMI $z$ score, children with moderate to severe OSA had a 4.2-fold increased risk of abnormal LV geometry compared with the reference group. This finding has important clinical implications as previous studies have found that in individuals with similar LV mass, altered LV geometry was associated with greater cardiovascular risk. ${ }^{31}{ }^{32}$ Altered LV diastolic filling is expected from concentric hypertrophy but there was no difference in mitral valve $\mathrm{E} / \mathrm{A}$ between the groups. The result may be accounted for by the sensitivity of early transmitral velocity (E) to loading condition, heart rate, as well as increase in LV mass. Transmitral flow in relation to tissue diastolic velocity Ea may be a better method of assessing diastolic function, ${ }^{27}{ }^{28}$ and $\mathrm{E} / \mathrm{e}^{\prime}$ was indeed shown to increase with OSA severity. This is in agreement with another study on LV function in children with OSA where the authors also demonstrated a dose dependent decrease in diastolic function with increased OSA severity. ${ }^{6}$ Adenotonsillectomy in children with OSA has been shown to improve growth, neurocognitive function and nocturnal enuresis. ${ }^{33}$ Well conducted studies that reported on the outcome of OSA treatment on cardiac findings are scarce. Görür et al showed improvement in RV dimension, LV end diastolic diameter and IVS assessed by ECHO after adenotonsillectomy. ${ }^{34}$ Unique to our study is the presence of a persistent OSA group that consisted of subjects who refused any treatment or did not have significant improvement after treatment. All children who underwent adenotonsillectomy had $a \geqslant 50 \%$ reduction in AHI, suggesting that adenotonsillectomy is indeed an effective treatment for childhood OSA (fig 1). On the other hand, only four out of nine children who received nasal steroid therapy had a $\geqslant 50 \%$ reduction in AHI. Significant improvements in RVMPI, IVSI, and $\mathrm{E} / \mathrm{e}^{\prime}$ comparing 6 month with baseline data were seen only in the improved OSA group, and indeed the change in RVMPI and $\mathrm{E} / \mathrm{e}^{\prime}$ was associated with a change in AHI. In the mild OSA group, five out of 18 untreated children had significant improvement in AHI, suggesting that some mild OSA cases may spontaneously improve with time. There may be a practical need for follow-up PSG for assessment of treatment efficacy and to monitor progress for those who do not receive treatment.

The severity of OSA in our sample population was milder than in other studies as they were recruited from the community. The results generated from our study are however, important as abnormal cardiac function and structure were already demonstrated in this community based cohort, emphasising the need to recognise OSA early in its progression. A proportion of OSA children did not return for repeat assessment at 6 months, which is one of our limitations and a common difficulty faced by paediatric researchers. ${ }^{35}$ On further analysis, there were no significant differences in anthropometric, PSG and ECHO measurements between those who did not return and untreated subjects. Cases with persistent OSA at follow-up had less severe disease at baseline than the improved OSA group which may be a potential bias. As treatment was not randomised, naturally parents of children with more severe OSA were more likely to opt for treatment and especially 
surgery. Although with milder disease at baseline, the ECHO parameters of the persistent OSA group did not improve with time. In contrast, significant improvements were seen following treatment in the improved OSA group, signifying genuine beneficial effects of intervention. The clinical significance of cardiac structural and functional changes described in this study, despite being shown in adult studies ${ }^{8}{ }^{30-32}$ to predict future clinical events, is not certain in children, and will require longer follow-up to better delineate their implications.

\section{CONCLUSIONS}

Childhood OSA is associated with RV and LV remodelling and dysfunction. Community based screening programmes may allow early detection and treatment of OSA that could potentially reverse myocardial dysfunction, remodelling and lessen the risk for future cardiovascular disease.

Acknowledgements: We are grateful for the support of all the staff of the Sleep Assessment Unit of Shatin Hospital, and the cooperation and participation of all the schools, children and their parents.

Funding: This study was supported by a grant (CUHK4161/02M) from the Research Grants Council of the Hong Kong Special Administrative Region, China.

Competing interests: None.

Ethics approval: The study was approved by the institutional ethics committee.

\section{REFERENCES}

1. Redline S, Tishler PV, Schluchter M, et al. Risk factors for sleep-disordered breathing in children. Associations with obesity, race, and respiratory problems. Am J Respir Crit Care Med 1999:159:1527-32.

2. Brouillette RT, Fernbach SK, Hunt CE. Obstructive sleep apnea in infants and children. J Pediatr 1982;100:31-40.

3. Brown $\mathbf{0 E}$, Manning SC, Ridenour B. Cor pulmonale secondary to tonsillar and adenoidal hypertrophy: management considerations. Int J Pediatr Otorhinolaryngol 1988:16:131-9.

4. Tal A, Leiberman A, Margulis G, et al. Ventricular dysfunction in children with obstructive sleep apnea: radionuclide assessment. Pediatr Pulmonol 1988;4:139-43.

5. Amin RS, Kimball TR, Bean JA, et al. Left ventricular hypertrophy and abnormal ventricular geometry in children and adolescents with obstructive sleep apnea. Am J Respir Crit Care Med 2002;165:1395-9.

6. Sanchez-Armengol A, Rodriquez-Puras MJ, Fuentes-Pradera MA, et al. Echocardiographic parameters in adolescents with sleep-related breathing disorders. Pediatr Pulmonol 2003;36:27-33.

7. Amin RS, Kimball TR, Kalra M, et al. Left ventricular function in children with sleepdisordered breathing. Am J Cardiol 2005;95:801-4

8. Redfield MM, Jacobsen SJ, Burnett JC, et al. Burden of systolic and diastolic ventricular dysfunction in the community: appreciating the scope of the heart failure epidemic. JAMA 2003;289:194-202.

9. Wang TJ, Evans JC, Benjamin EJ, et al. Natural history of asymptomatic left ventricular systolic dysfunction in the community. Circulation 2003;108:977-82

10. Li AM, Cheung A, Chan $D$, et al. Validation of a questionnaire instrument for prediction of obstructive sleep apnea in Hong Kong Chinese children. Pediatr Pulmonol 2006:41:1153-60.

11. Leung SS, Cole TJ, Tse LY, et al. Body mass index reference curves for Chinese children. Ann Hum Biol 1998;25:169-74.
12. Berlucchi M, Salsi D, Valetti L, et al. The role of mometasone furoate aqueous nasal spray in the treatment of adenoidal hypertrophy in the pediatric age group: preliminary results of a prospective, randomized study. Pediatrics 2007;119:e1392-7.

13. American Academy of Sleep Medicine. The international classification of sleep disorders, 2nd Edn, diagnostic and coding manual. Westchester, IL: American Academy of Sleep Medicine, 2005:56-9.

14. Devereux RB, Alonso DR, Lutas EM, et al. Echocardiographic assessment of left ventricular hypertrophy: comparison to necropsy findings. Am J Cardiol 1986;57:450-8.

15. Daniels SR, Kimball TR, Morrison JA, et al. Indexing left ventricular mass to account for differences in body size in children and adolescents without cardiovascular disease. Am J Cardiol 1995;76:699-701.

16. de Simone G, Daniels SR, Kimball TR, et al. Evaluation of concentric left ventricular geometry in humans: evidence for age-related systematic underestimation. Hypertension 2005;45:64-8.

17. Ganau A, Devereux RB, Roman MJ, et al. Patterns of left ventricular hypertrophy and geometric remodeling in essential hypertension. J Am Coll Cardiol 1992;19:1550-8.

18. Pendlebury ST, Pépin JL, Veale D, et al. Natural evolution of moderate sleep apnoea syndrome: significant progression over a mean of 17 months. Thorax 1997;52:872-8.

19. Amin R, Somers VK, McConnell K, et al. Activity-adjusted 24-hour ambulatory blood pressure and cardiac remodeling in children with sleep disordered breathing. Hypertension 2008;51:84-91.

20. Qureshi N, Joyce JJ, Qi N, et al. Right ventricular abnormalities in sickle cell anemia: evidence of a progressive increase in pulmonary vascular resistance. $J$ Pediatr 2006;149:23-7.

21. Okin PM, Wachtell K, Devereux RB, et al. Regression of electrocardiographic left ventricular hypertrophy and decreased incidence of new-onset atrial fibrillation in patients with hypertension. JAMA 2006;296:1242-8.

22. Okin PM, Devereux RB, Jern S, et al; LIFE Study Investigators. Regression of electrocardiographic left ventricular hypertrophy during antihypertensive treatment and the prediction of major cardiovascular events. JAMA 2004;292:2343-9.

23. Sanner BM, Konermann M, Sturm A, et al. Right ventricular dysfunction in patients with obstructive sleep apnoea syndrome. Eur Respir J 1997;10:2079-83.

24. Guidry UC, Mendes LA, Evans JC, et al. Echocardiographic features of the right heart in sleep-disordered breathing: the Framingham Heart Study. Am J Respir Crit Care Med 2001;164:933-8.

25. Dursunoglu N, Dursunoglu D, Kilic M. Impact of obstructive sleep apnea on right ventricular global function: sleep apnea and myocardial performance index. Respiration 2005;72:278-84.

26. Wing YK, Hui SH, Pak WM, et al. A controlled study of sleep related disordered breathing in obese children. Arch Dis Child 2003;88:1043-7.

27. Wong CY, O'Moore-Sullivan T, Leano $R$, et al. Alterations of left ventricular myocardial characteristics associated with obesity. Circulation 2004;110:3081-7.

28. Peterson LR, Waggoner AD, Schechtman KB, et al. Alterations in left ventricular structure and function in young healthy obese women: assessment by echocardiography and tissue Doppler imaging. J Am Coll Cardiol 2004:43:1399-404.

29. Hunt CE, Brouillette RT. Abnormalities of breathing control and airway maintenance in infants and children as a cause of cor pulmonale. Pediatr Cardiol 1982;3:249-56.

30. Muiesan ML, Salvetti M, Paini A, et al. Inappropriate left ventricular mass changes during treatment adversely affects cardiovascular prognosis in hypertensive patients. Hypertension 2007:49:1077-83.

31. Verdecchia $\mathbf{P}$, Schillaci G, Borgioni $C$, et al. Prognostic value of left ventricular mass and geometry in systemic hypertension with left ventricular hypertrophy. Am J Cardiol 1996; 78:197-202.

32. Krumholz HM, Larson M, Levy D. Prognosis of left ventricular geometric patterns in the Framingham Heart Study. J Am Coll Cardiol 1995;25:879-84.

33. Leiberman A, Stiller-Timor L, Tarasiuk A, et al. The effect of adenotonsillectomy on children suffering from obstructive sleep apnea syndrome (OSAS): the Negev perspective. Int J Pediatr Otorhinolaryngol 2006;70:1675-82.

34. Görür K, Doven 0, Unal M, et al. Preoperative and postoperative cardiac and clinical findings of patients with adenotonsillar hypertrophy. Int J Pediatr Otorhinolaryngol 2001:59:41-6.

35. Turner SW, le Souef PN. Is patient dropout from a longitudinal study of lung function predictable and reversible? Pediatr Pulmonol 2003;35:29-33. 